2 December 2018

\title{
Electronic and center of mass transitions driven by Laguerre-Gaussian beams
}

\author{
A Alexandrescu ${ }^{1} \dagger$, E Di Fabrizio ${ }^{2}$ and D Cojoc ${ }^{1,2}$ \\ ${ }^{1} \mathrm{CCO}$ - Optoelectronics Research Center and Department of Technology and \\ Reliability, "Politehnica" University of Bucharest, RO-61071, Romania \\ ${ }^{2}$ TASC-INFM @ Elettra Sincrotrone LILIT - Beam line Basovizza-Trieste, \\ IT-34012, Italy
}

\begin{abstract}
We derive the interaction Hamiltonian of a Laguerre-Gaussian beam with a simple atomic system, under the assumption of a small spread of the center of mass wave function in comparison with the waist of the Laguerre-Gaussian beam. The center of mass motion of the atomic system is taken into account. Using the properties of regular spherical harmonics the internal and center of mass coordinates are separated without making any multipolar expansion. Then the selection rules of the internal and of the center of mass motion transitions follow immediately. The influence of the winding number of the Laguerre-Gaussian beams on the selection rules and transition probability of the center of mass motion is discussed.

PACS numbers: $32.80 . \mathrm{Lg}, 42.50 . \mathrm{Vk}$
\end{abstract}

\section{Introduction}

The exploration of the orbital angular momentum (OAM) of the electromagnetic field has been impelled by the work of Allen et al. [1] who pointed out that the LaguerreGaussian (LG) modes carries a well defined amount of OAM per unit energy. Since then the OAM properties of the radiation have gained much interest, the concepts of OAM being applied in several fields of optics. Ref. [2] contains a collection of articles devoted or in connection to the OAM of the radiation field. We can take advantage of the OAM of the electromagnetic field to study the entanglement in a $n$-dimensional Hilbert space by spontaneous parametric down-conversion [3]. The interaction of LG modes with atoms as point particles has been studied [4] and the transfer of the light OAM to atoms was observed in a four-wave mixing process [5]. Further, by taking into account the center of mass (CM) motion of the atomic system, it was shown that radiation field endowing OAM may entangle the internal and external OAM of the atom [6], and the field OAM is imparted between the internal and external motion of the atomic system [7, 8. The aim of this work is to investigate how the selection rules and the probability

$\dagger$ Author to whom any correspondence should be addressed: aalexandrescu@tehfi.pub.ro 
of transitions of the atomic systems change under the influence of an LG beam, taking into account th CM motion of the atomic system.

\section{The atom-radiation system}

We consider here the simplest atomic system, formed by a positive charge $+e$ of mass $m_{n}$ and a negative charge $-e$ of mass $m_{e}$, both of them spinless. The Hamiltonian of the system formed by the atom and the electromagnetic field is given by $H=H_{0}+H_{\text {int }}$, where $H_{0}$ is the unperturbed Hamiltonian of the atom and $H_{\mathrm{int}}$ is the interaction Hamiltonian of the atom with the electromagnetic field, specified ahead in the text.

The CM coordinate of the atomic system will be given by $\mathbf{R}=\left(m_{\mathrm{e}} \mathbf{r}_{\mathrm{e}}+m_{\mathrm{n}} \mathbf{r}_{\mathrm{n}}\right) / m_{\mathrm{t}}$, $m_{\mathrm{t}}=m_{\mathrm{e}}+m_{\mathrm{n}}$ being the total mass, and their relative (internal) coordinate by $\mathbf{r}=\mathbf{r}_{\mathrm{e}}-\mathbf{r}_{\mathrm{n}}$, where $\mathbf{r}_{\mathrm{e}}$ and $\mathbf{r}_{\mathrm{n}}$ represent the particles coordinates. Further, we assume that the atom state can be written as product of the CM wave function and the internal, i.e. electronic, wavefunction $\Upsilon(\mathbf{R}, \mathbf{r})=\Psi_{R}(\mathbf{R}) \Psi(\mathbf{r})$. The atomic system is considered trapped in a two dimensional harmonic potential, and its normalized wavefunction written in cylindrical coordinates has the form:

$$
\Psi_{R}(\mathbf{R})=\frac{1}{2 \pi} G_{N, M}\left(\frac{R_{\perp}}{w_{R}}\right) \exp \left[\mathrm{i}\left(K R_{z}+M \Phi\right)\right]
$$

where $G_{N, M}(x)=\mathcal{N} x^{|M|} L_{n^{-}}^{|M|}\left(x^{2}\right) \exp \left(-x^{2} / 2\right), L_{p}^{l}(x)$ being the generalized Laguerre

polynomial, and $w_{R}$ describes the spread of the CM wavefunction. $\mathcal{N}=\sqrt{2 n^{-} ! / n^{+}}$! $w_{R}$ is the normalization constant, with $n^{ \pm}=(N \pm|M|) / 2$. The quantum number $N$ gives the energy of the harmonic oscillator $E_{R}=\hbar^{2}(N+1) /\left(w_{R}^{2} m_{\mathrm{t}}\right)$, and $M$ gives the amount of angular momentum carried by the CM motion $M \hbar$. The internal wave function is given in terms of spherical harmonics $Y_{l}^{m}$ :

$$
\Psi(\mathbf{r})=F_{n, l}(r) Y_{l}^{m}(\theta, \varphi),
$$

where $F_{n, l}(r)$ gives the radial dependence.

We consider an electromagnetic field, in the paraxial approximation, described by a LG beam of waist $w_{0}$, having no off-axis radial nodes, propagating along the $z$ axis, and normalized according to $\int \mathrm{d}^{2} r|E(\mathbf{r})|^{2}=A$. The normalization constant $A$ is chosen in such way that it is independent of the winding number $l$. Under the assumption that the spread of the CM wave function is smaller in comparison with the waist of the LG beam, the electric field reads as [6]:

$$
\mathbf{E}(\mathbf{r}, t)=\frac{\mathbf{E}_{0}}{\sqrt{|l| !}}\left(\frac{r_{\perp}}{w_{0}}\right)^{|l|} \exp [\mathrm{i}(l \varphi+k z-\omega t)] .
$$

Next, we write the electromagnetic field in terms of regular solid spherical harmonics $\mathcal{R}_{l}^{m}$ :

$$
\mathbf{E}(\mathbf{r}, t)=(-1)^{\frac{l+|l|}{2}} 2^{|l|} \sqrt{|l| !} \mathbf{E}_{0} \mathcal{R}_{|l|}^{l}\left(\frac{\mathbf{r}_{\perp}}{w_{0}}\right) \exp [\mathrm{i}(k z-\omega t)],
$$

where $\mathbf{E}_{0}$ is the polarization vector of the electric field, and $\mathcal{R}_{l}^{m}\left(\mathbf{r}_{\perp}\right)=\mathcal{C}_{l}^{m} r^{l} Y_{l}^{m}(\theta=$ $\pi / 2, \varphi)$ is the regular solid spherical harmonic [9], with $\mathcal{C}_{l}^{m}=[4 \pi /(2 l+1) /(l-m) ! /(l+$ 
$m) !]^{1 / 2}$. For simplicity, the symmetry axis of the CM motion is considered to be the same as the axis of the electromagnetic LG mode. In the subsequent calculations the electromagnetic field is treated classically.

\section{The interaction Hamiltonian}

We now focus on the interaction Hamiltonian which plays the central role in revealing the influence of the winding number $l$ on system transitions. The structure of the electromagnetic field (44), i.e., in terms of spherical harmonics, is straightway imprinted to the interaction Hamiltonian using the Power-Zienau-Wooley scheme [10]:

$$
H_{\text {int }}=-\int \mathrm{d}^{3} r \mathcal{P}(\mathbf{r}) \cdot \mathbf{E}(\mathbf{r}, t)+\text { h.c. },
$$

where $\mathcal{P}(\mathbf{r})$ represent the polarization density. Writing the polarization density as a closed integral

$$
\mathcal{P}(\mathbf{r})=\sum_{\alpha=n, e} e_{\alpha}\left(\mathbf{r}_{\alpha}-\mathbf{R}\right) \int_{0}^{1} \mathrm{~d} \lambda \delta\left[\left(\mathbf{r}-\mathbf{R}-\lambda\left(\mathbf{r}_{\alpha}-\mathbf{R}\right)\right)\right]
$$

and introducing it in relation (5), the interaction Hamiltonian is expressed as follows 7]:

$H_{\text {int }}=\frac{e}{m_{\mathrm{t}}} \mathbf{r} \cdot \int_{0}^{1} \mathrm{~d} \lambda\left\{m_{\mathrm{n}} \mathbf{E}\left(\mathbf{R}+\lambda \frac{m_{\mathrm{n}}}{m_{\mathrm{t}}} \mathbf{r}, t\right)+m_{\mathrm{e}} \mathbf{E}\left(\mathbf{R}-\lambda \frac{m_{\mathrm{e}}}{m_{\mathrm{t}}} \mathbf{r}, t\right)\right\}+$ h.c. $=H_{\text {int }}^{(1)}+H_{\mathrm{int}}^{(2)}$,

where $H_{\text {int }}^{(1)}$ and $H_{\text {int }}^{(2)}$ refers to the terms given by $m_{\mathrm{n}} \mathbf{E}\left(\mathbf{R}+\lambda \frac{m_{\mathrm{n}}}{m_{\mathrm{t}}} \mathbf{r}, t\right)+$ h.c. and $m_{\mathrm{e}} \mathbf{E}\left(\mathbf{R}+\lambda \frac{m_{\mathrm{e}}}{m_{\mathrm{t}}} \mathbf{r}, t\right)+$ h.c., respectively.

Using the expression of electromagnetic field (4) in terms of regular spherical harmonics, we can separate the CM and electronic coordinates, $\mathbf{R}$ and $\mathbf{r}$, respectively, by the translation property of regular spherical harmonics $[9]$ :

$$
\mathcal{R}_{l}^{m}(\mathbf{x} \pm \mathbf{y})=\sum_{l^{\prime}=0}^{l} \sum_{m^{\prime}=-l^{\prime}}^{l^{\prime}}( \pm)^{l^{\prime}} \mathcal{R}_{l^{\prime}}^{m^{\prime}}(\mathbf{x}) \mathcal{R}_{l-l^{\prime}}^{m-m^{\prime}}(\mathbf{y})
$$

and the electromagnetic field will read as:

$$
\begin{aligned}
\mathbf{E}\left(\mathbf{R}+\lambda \frac{m_{\mathrm{n}}}{m_{\mathrm{t}}} \mathbf{r}\right)= & (-1)^{\frac{l+|l|}{2}} 2^{|l|} \sqrt{|l| !} \mathbf{E}_{0} \exp \left[\mathrm{i} k\left(R_{z}+\lambda \frac{m_{\mathrm{n}}}{m_{\mathrm{t}}} z\right)\right] \sum_{l^{\prime}=0}^{|l|} \sum_{m^{\prime}=-l^{\prime}}^{l^{\prime}} \mathcal{C}_{l^{\prime}}^{m^{\prime}} \mathcal{C}_{|l|-l^{\prime}}^{l-m^{\prime}} \\
& \times\left(\lambda \frac{m_{\mathrm{n}} r_{\perp}}{m_{\mathrm{t}} w_{0}}\right)^{l^{\prime}}\left(\frac{R_{\perp}}{w_{0}}\right)^{|l|-l^{\prime}} Y_{l^{\prime}}^{m^{\prime}}\left(\theta=\frac{\pi}{2}, \varphi\right) Y_{|l|-l^{\prime}}^{l-m^{\prime}}\left(\Theta=\frac{\pi}{2}, \Phi\right)
\end{aligned}
$$

From the properties of the spherical harmonics functions $Y_{l}^{m}(\theta, \varphi)$ we know that in the case $\theta=\pi / 2$, the functions takes on non-zero values only if $l+m$ is an even number. The indices of the spherical harmonics functions corresponding to the internal and CM coordinates, in the r.h.s of relation (9), are $\left(l^{\prime}, m^{\prime}\right)$ and $\left(|l|-l^{\prime}, l-m^{\prime}\right)$, respectively. This implies that when $l^{\prime}+m^{\prime}$, also $|l|-l^{\prime}+l-m^{\prime}$ is an even number, so the spherical functions takes on non-zero values at the same time and the double sum does not become zero. 
Introducing the explicit values of the functions $Y_{l}^{m}(\theta=\pi / 2, \phi)$ into equation (9) we get:

$$
\begin{aligned}
\mathbf{E}(\mathbf{R} & \left.+\lambda \frac{m_{\mathrm{n}}}{m_{\mathrm{t}}} \mathbf{r}\right)=2^{|l|} \sqrt{|l| !} \mathbf{E}_{0} \exp \left[\mathrm{i} k\left(R_{z}+\lambda \frac{m_{\mathrm{n}}}{m_{\mathrm{t}}} z\right)\right] \sum_{l^{\prime}=0}^{|l|} \sum_{m^{\prime}=-l^{\prime}}^{l^{\prime}}\left(\lambda \frac{m_{\mathrm{n}} r_{\perp}}{m_{\mathrm{t}} w_{0}}\right)^{l^{\prime}}\left(\frac{R_{\perp}}{w_{0}}\right)^{|l|-l^{\prime}} \\
& \times \frac{\exp (\mathrm{i} m \varphi) \exp \left[\mathrm{i}\left(l-m^{\prime}\right) \Phi\right]}{\left(l^{\prime}-\left|m^{\prime}\right|\right) ! !\left(l^{\prime}+\left|m^{\prime}\right|\right) ! !\left(|l|-l^{\prime}-\left|l-m^{\prime}\right|\right) ! !\left(|l|-l^{\prime}+\left|l-m^{\prime}\right|\right) ! !} \delta_{l^{\prime}+m^{\prime}, \text { even }}
\end{aligned}
$$

where $n$ !! stands for the double factorial. Let us analyze the influence of the double factorial $\left(|l|-l^{\prime}+\left|l-m^{\prime}\right|\right)$ !! on the double sum appearing in the above relation. Taking into account that $\left|m^{\prime}\right| \leq l^{\prime}$ and $l^{\prime}+m^{\prime}$ must be even, there are two possible situations:

(i) $l>0$ leads to $|l|-l^{\prime}+\left|l-m^{\prime}\right|=-l^{\prime}+m^{\prime}$ which takes on either zero or negative values. The properties of the double factorial tell us that $n ! != \pm \infty$ unless $n \geq 0$. The case $-l^{\prime}+m^{\prime}<0$ is ruled out, and we are left with $m^{\prime}=l^{\prime}$.

(ii) $l<0$ leads to $|l|-l^{\prime}+\left|l-m^{\prime}\right|=-l^{\prime}-m^{\prime}$ also taking on either zero or negative values. Performing a similar inference to previous case, the only valid possibility is $m^{\prime}=-l^{\prime}$.

Summarizing the conclusion of (ii) and (iii), the double sum is reduced to a simple sum where $m^{\prime}=\operatorname{sgn}(l) l^{\prime}$. The equation (10) becomes

$$
\begin{aligned}
& \mathbf{E}\left(\mathbf{R}+\lambda \frac{m_{\mathrm{n}}}{m_{\mathrm{t}}} \mathbf{r}\right)=2^{|l|} \sqrt{|l| !} \mathbf{E}_{0} \exp \left[\mathrm{i} k\left(R_{z}+\lambda \frac{m_{\mathrm{n}}}{m_{\mathrm{t}}} z\right)\right] \sum_{l^{\prime}=0}^{|l|}\left(\lambda \frac{m_{\mathrm{n}} r_{\perp}}{m_{\mathrm{t}} w_{0}}\right)^{l^{\prime}}\left(\frac{R_{\perp}}{w_{0}}\right)^{|l|-l^{\prime}} \\
& \times \frac{\exp \left[i \operatorname{sgn}(l) l^{\prime} \varphi\right] \exp \left[i \operatorname{sgn}(l)\left(|l|-l^{\prime}\right) \Phi\right]}{\left(2 l^{\prime}\right) ! !\left[2\left(|l|-l^{\prime}\right)\right] ! !}
\end{aligned}
$$

In the above relation, one may change the internal coordinate $r_{\perp}$ to $r \sin \theta$ in order to give the interaction Hamiltonian in terms of spherical harmonics. Replacing the expression of the plane wave by its expansion into spherical harmonics $\exp (\mathrm{i} k r \cos \theta)=$ $\sum_{p=0}^{\infty} \mathrm{i}^{p} \sqrt{4 \pi(2 p+1)} j_{p}(k r) Y_{p}^{0}(\theta)$, where $j_{\nu}(x)$ is the spherical Bessel function of the first kind of order $\nu$, the integration over $\lambda$ is performed and the interaction Hamiltonian $H_{\text {int }}^{(1,2)}$ reads as:

$$
\begin{aligned}
H_{\mathrm{int}}^{(1,2)}=( \pm) & \frac{2 \pi^{2} e w_{0}}{\sqrt{3}}\left(\frac{4}{k w_{0}}\right)^{|l|+1} \sqrt{|l| !} \exp \left(\mathrm{i} k R_{z}\right) \\
& \times \sum_{p=0}^{\infty} \sum_{l^{\prime}=0}^{|l|} \sum_{\sigma=0, \pm 1} \epsilon_{\sigma}\left[\frac{(2 p+1)}{\Gamma\left(2 l^{\prime}+2\right)}\right]^{\frac{1}{2}} \frac{\mathrm{i}^{p+l^{\prime}(1+\operatorname{sgn}(l))}}{\left(1+p+l^{\prime}\right) \Gamma\left(p+\frac{3}{2}\right) \Gamma\left(|l|-l^{\prime}+1\right)} \\
& \times\left(\frac{k R_{\perp}}{4}\right)^{|l|-l^{\prime}}\left( \pm k r \frac{m_{\mathrm{n}, \mathrm{e}}}{2 m_{\mathrm{t}}}\right)^{l^{\prime}+p+1} Y_{l^{\prime}}^{\mathrm{sgn}(l) l^{\prime}}(\theta, \varphi) Y_{1}^{\sigma}(\theta, \varphi) Y_{p}^{0}(\theta) e^{\mathrm{i} \operatorname{sgn}(l)\left(|l|-l^{\prime}\right) \Phi} \\
& \times{ }_{1} F_{2}\left(\frac{p+l^{\prime}+1}{2} ; p+\frac{3}{2}, \frac{p+l^{\prime}+3}{2} ;-\left(k r \frac{m_{\mathrm{n}, \mathrm{e}}}{2 m_{\mathrm{t}}}\right)^{2}\right)+\text { h.c. },
\end{aligned}
$$

where $\mathbf{r} \cdot \mathbf{E}_{0}$ was replaced by $r \sqrt{4 \pi / 3} \sum_{\sigma=0, \pm 1} \epsilon_{\sigma} Y_{1}^{\sigma}(\theta, \varphi), \sigma$ being associated with the polarization of the electric field, $\epsilon_{ \pm 1}= \pm\left(E_{x} \pm \mathrm{i} E_{y}\right) / \sqrt{2}$ and $\epsilon_{0}=E_{z}$. However, in the frame of paraxial approximation, the $E_{z}$ component of the electromagnetic field has a small contribution to the total electric field. The latter one can be given, to a good approximation, in terms of the transverse electric field [11]. 
In relation (12), since $p$ runs from zero to infinity and the hypergeometric function ${ }_{1} F_{2}$ consists of an infinitely series, it follows that all multipole transitions are excited. Nevertheless, the factor $r^{l^{\prime}+p+1}$ allows us to consider the first and the second transitions, while keeping only the first term, i.e., the constant one, of the hypergeometric function ${ }_{1} F_{2}: l^{\prime}+p+1=1$ gives the dipole transition, $l^{\prime}+p+1=2$ gives the quadrupole transition. We note that in the dipole approximation, there is no transfer of the field OAM to the electronic motion, our result being consistent with the concluding remark of Ref. [7.

\section{Selection rules and transition probabilities}

The selection rules of a certain transition are obtained by the transition matrix element between the final and initial state $\mathcal{M}_{i \rightarrow f}^{(1,2)}=\left\langle\Upsilon_{f}\left|H_{\text {int }}^{(1,2)}\right| \Upsilon_{i}\right\rangle$. Putting together the expression of the interaction Hamiltonian (12) and of the atomic wave function (11)(2), the transition matrix element is written as:

$$
\begin{aligned}
\mathcal{M}_{i \rightarrow f}^{(1,2)}=( \pm) & \frac{2 \pi^{2} w_{0} e}{\sqrt{3}} \sqrt{|l| !}\left(\frac{4}{k w_{0}}\right)^{|l|+1} \delta\left(k+K_{i}-K_{f}\right) \\
& \times \sum_{L=0}^{\infty} \sum_{l^{\prime}=0}^{|l|} \sum_{\sigma=0, \pm 1} \epsilon_{\sigma}\left[\frac{(2 p+1)}{\Gamma\left(2 l^{\prime}+2\right)}\right]^{\frac{1}{2}} \frac{\mathrm{i}^{p+l^{\prime}(1+\operatorname{sgn}(l))}}{\left(l^{\prime}+p+1\right) \Gamma(p+3 / 2) \Gamma\left(|l|-l^{\prime}+1\right)} \\
& \times\left\langle G_{N_{f}, M_{f}}\left|\left(\frac{k R_{\perp}}{4}\right)^{|l|-l^{\prime}}\right| G_{N_{i}, M_{i}}\right\rangle \delta_{\left(M_{f}-M_{i}\right), \operatorname{sgn}(l)\left(|l|-l^{\prime}\right)} \\
& \times\left\langle F_{n_{f}, l_{f}}\left|\left( \pm k r \frac{m_{\mathrm{n}, \mathrm{e}}}{2 m_{\mathrm{t}}}\right)^{l^{\prime}+p+1}{ }_{1} F_{2}\left(\frac{p+l^{\prime}+1}{2} ; p+\frac{3}{2}, \frac{p+l^{\prime}+3}{2} ;-\left(k r \frac{m_{\mathrm{n}, \mathrm{e}}}{2 m_{\mathrm{t}}}\right)^{2}\right)\right| F_{n_{i}, l_{i}}\right\rangle \\
& \times\left\langle Y_{l_{f}}^{m_{f}}\left|Y_{l^{\prime}}^{\mathrm{sgn}(l) l^{\prime}} Y_{1}^{\sigma} Y_{p}^{0}\right| Y_{l_{i}}^{m_{i}}\right\rangle .
\end{aligned}
$$

In relation (13) the Dirac delta function and Kronecker delta symbol express the conservation of the axial component of the CM momentum and the conservation of CM OAM, respectively. The bracket symbols designate the integral over the radial components $R_{\perp}$ and $r$, and over the internal solid angle element $\mathrm{d}(\cos \theta) \mathrm{d} \varphi$, respectively. The transition probabilities are proportional to the integrals over the radial coordinates $R_{\perp}$ and $r$, being directly influenced by the absolute value of the winding number $l$.

The selection rules which governs the electronic-type transitions are given by the integral involving the spherical harmonics in relation (13). Using the addition theorem of angular momentum, the spherical harmonics can be coupled and the final result expressed in terms of Clebsch-Gordan coefficients. The parity selection rules can be easily determined taking into account the properties of spherical harmonics, the transition being allowed only when $l_{f}+l_{i}+l^{\prime}+p+1$ is an even number. From Table 1 one can see that the selection rules of the dipole and quadrupole transition are unchanged, as expected. The $z$ component of the angular momentum of the entire system is conserved, i.e., the relation $\Delta m+\Delta M=l+\sigma$ is always fulfilled [8]. Here, since we are in the paraxial approximation, the polarization of the electromagnetic field determines changes only in the internal magnetic quantum number $m$ of the atom, but 
Electronic and center of mass transitions

\begin{tabular}{|c|c|c|c|c|c|c|c|c|}
\hline \multirow[t]{2}{*}{ Transitions driven by field OAM } & \multirow{2}{*}{\multicolumn{2}{|c|}{$L \quad l$}} & \multirow[t]{2}{*}{$\operatorname{sgn}(l)$} & \multirow[t]{2}{*}{$\Delta l$} & \multicolumn{3}{|c|}{$\Delta m$} & \multirow[t]{2}{*}{$\Delta M$} \\
\hline & & & & & -1 & 0 & +1 & \\
\hline CM transition & 0 & 0 & & \pm 1 & -1 & 0 & +1 & $l$ \\
\hline CM transition & 1 & 0 & & $0, \pm 2$ & -1 & 0 & +1 & $l$ \\
\hline CM and electronic quadrupole & 0 & 1 & +1 & $0, \pm 2$ & 0 & 1 & +2 & $l-1$ \\
\hline CM and electronic quadrupole & 0 & 1 & -1 & $0, \pm 2$ & -2 & -1 & 0 & $-|l|+1$ \\
\hline
\end{tabular}

Table 1. Selection rules of transitions induced by an LG beam: $(\Delta l, \Delta m)$ and $\Delta M$ are the changes of the electronic-type quantum numbers and of the CM OAM, respectively. The numbers under column of $\Delta m$ correspond to different polarizations $\sigma$ of the electromagnetic field.

beyond paraxial approximation this is no longer valid [12. In relation (13) as $l^{\prime}$ runs from zero to $|l|$ the OAM of the electromagnetic field is imparted to the CM motion and to the electronic-type motion. The maximum value of the transferred angular momentum either to the CM motion or to the electronic-type motion does not exceed $|l|$. This feature is a peculiarity due to the radial dependence of the electromagnetic field, assumed in relation (31); however, in general, no bound on the value of the angular momentum transferred is set [7]. Although the selection rules are not directly governed by the winding number $l$ of the LG beam, they are sensitive to the sign of $l$ as one can see from Table 1 in the case of the quadrupole transitions with $p=0$ and $l^{\prime}=1$.

The influence of the winding number $l$ on the transition probability can be analyzed by performing the radial integral over the $\mathrm{CM}$ coordinate $R_{\perp}$ in formula (13). Considering the initial and final CM states of the form (11), the integral is given by [13]:

$$
\begin{aligned}
\left\langle G_{N_{f}, M_{f}}\left|\left(\frac{k R_{\perp}}{4}\right)^{|l|-l^{\prime}}\right| G_{N_{i}, M_{i}}\right\rangle=\frac{w_{R}^{2}}{2} \mathcal{N}_{i} \mathcal{N}_{f}\left(\frac{k w_{R}}{4}\right)^{|l|-l^{\prime}} \\
\quad \times \int_{0}^{\infty} \mathrm{d} x x^{\left(\left|M_{i}\right|+\left|M_{f}\right|+|l|-l^{\prime}\right) / 2} e^{-x} L_{n_{i}^{-}}^{\left|M_{i}\right|}(x) L_{n_{f}^{-}}^{\left|M_{f}\right|}(x) \\
=\left(\frac{k w_{R}}{4}\right)^{|l|-l^{\prime}} \frac{\left(1+\left|M_{i}\right|\right)_{n_{i}^{-}}\left(\frac{\left|M_{f}\right|-\left|M_{i}\right|-|l|+l^{\prime}}{2}\right)_{n_{f}^{-}} \Gamma\left(\frac{\left|M_{i}\right|+\left|M_{f}\right|+|l|-l^{\prime}}{2}+1\right)}{\left(n_{i}^{-} ! n_{f}^{-} ! n_{i}^{+} ! n_{f}^{+} !\right)^{1 / 2}} \\
\quad \times{ }_{3} F_{2}\left(-n_{i}^{-}, \frac{\left|M_{i}\right|+\left|M_{f}\right|+|l|-l^{\prime}}{2}+1, \frac{\left|M_{i}\right|-\left|M_{f}\right|+|l|-l^{\prime}}{2}+1 ; 1+\left|M_{i}\right|, \frac{\left|M_{i}\right|-N_{f}+|l|-l^{\prime}}{2}+1 ; 1\right),
\end{aligned}
$$

with $M_{f}=M_{i}+\operatorname{sgn}(l)\left(|l|-l^{\prime}\right)$. Depending on the sign of the product $l M_{i}$, the relation (14) may be further simplified.

Next, we will focus on the CM transitions due to the photon absorption in the dipole interaction, $p=l^{\prime}=0$, and in the quadrupole interaction, with one OAM unit transferred to the electronic motion, $p=0$ and $l^{\prime}=1$. The dependence of the CM transition probability $P_{C M}$ on $l, l^{\prime}$ and $w_{R} / w_{0}$ is the form

$$
P_{C M} \propto\left|\frac{i^{l^{\prime}(1+\operatorname{sgn}(l))}}{\left(1+l^{\prime}\right) \Gamma\left(|l|-l^{\prime}+1\right)}\left(\frac{4}{k w_{0}}\right)^{|l|+1}\left[\frac{|l| !}{\Gamma\left(2 l^{\prime}+2\right)}\right]^{\frac{1}{2}}\left\langle G_{N_{f}, M_{f}}\left|\left(\frac{k R_{\perp}}{4}\right)^{|l|-l^{\prime}}\right| G_{N_{i}, M_{i}}\right\rangle\right|^{2}
$$


The selection rules of CM transitions are given by (i) the properties of the 1Dharmonic oscillator (through the radial integral (14)) and (ii) angular momentum conservation law (through the variable $\Phi$ ), which fixes the quantum numbers $N_{f}$ and $M_{f}$. In figure 1 we plot the transition probability $P_{C M}$ as function of $N_{f}$ for $l=2$ (left) and $l=3$ (right). In figure 1a, the CM selection rules are $\Delta N=N_{f}-N_{i}=0, \pm 2$ (with the electronic transition of the dipole type, $l^{\prime}=0$ ), and $\Delta N= \pm 1$ (with the electronic transition of the quadrupole type, $l^{\prime}=1$ ). In figure $1 \mathrm{~b}$, for $l=3$, the number of the final CM states which can be addressed is larger in comparison with figure 1a, due to the multipole CM transition $|l|-l^{\prime}=2,3$.

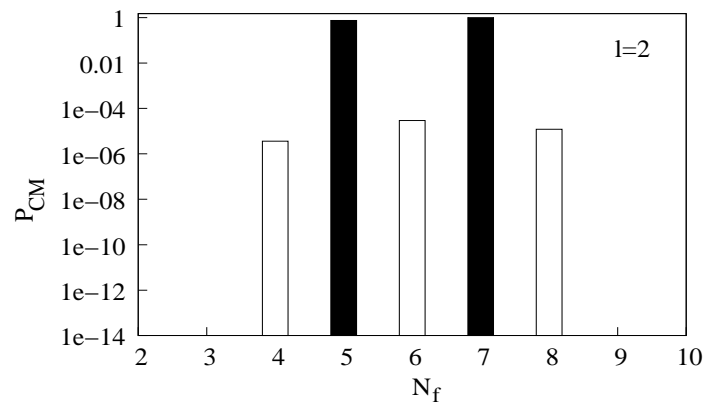

(a)

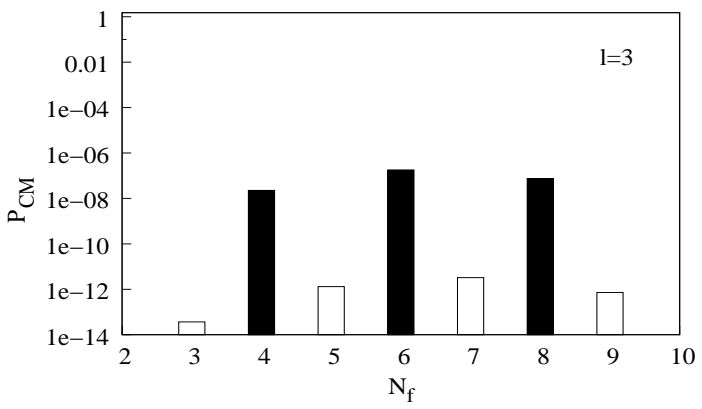

(b)

Figure 1. The CM transition probability $P_{C M}$ as function of the energy quantum number $N_{f}$, for $N_{i}=6, M_{i}=0, w_{R} / w_{0}=10^{-4}, l=2$ (left) and $l=3$ (right). One can see how the interaction type, i.e. dipole or quadrupole, changes with $l$ for the final states. The white and black boxes correspond to CM transitions due to the electronic dipole $\left(l^{\prime}=0\right)$ and quadrupole $\left(l^{\prime}=1\right)$ interaction, respectively. The transition probabilities are normalized to the the value corresponding to $N_{f}=6$ in the left figure.

The quantum numbers of energy $N$ and of the angular momentum $M$, appearing in relation (11) must be of the same parity (this comes from the fact that the wavefunction (11) describes two uncoupled 1D-harmonic oscillators with the quantum numbers $n_{x}$ and $n_{y}$, and from here follows $N=n_{x}+n_{y}$ and $\left.M=n_{x}-n_{y}\right)$. When one considers only the interaction in the leading orders, the difference in the quantum number $M_{f}$ will be $M_{f}^{(\mathrm{d})}-M_{f}^{(\mathrm{q})}= \pm 1$, where (d) and (q) designates the dipole and quadrupole interaction, respectively. Therefore the energy number of final states reached by dipole interaction will have opposite parity in comparison with that one of final states reached by quadrupole interaction, see also figure 1. It follows that we can not address using only one LG beam a specific state by both dipole and quadrupole interaction. To enable both interaction types one may use superpositions of LG beams having different winding numbers $l$, e.g. by suitable placed non-axial vortices [14].

From figure 11 one sees that the CM transitions occurring due to the electronic dipole interaction are about $10^{6}$ times weaker than those occurring due to the electronic quadrupole interaction. However, when one calculates the overall transition probability, i.e., electronic and CM, the difference between these two transition types will decrease 
to about $10^{2}$ times, because the electronic quadrupole transitions are about $10^{8}$ times more unlikely that the electronic dipole transitions.

In figure 2 we plot the CM transition probability (15) as a function of the winding number $l$ and of the ratio $w_{R} / w_{0}$, between the initial $N_{i}=6, M_{i}=0$ and final $N_{f}=12$ state. The transitions are driven by the dipole (quadrupole) interaction for $l$ even (odd). Introducing the expression of the matrix element (14) into relation (15) the

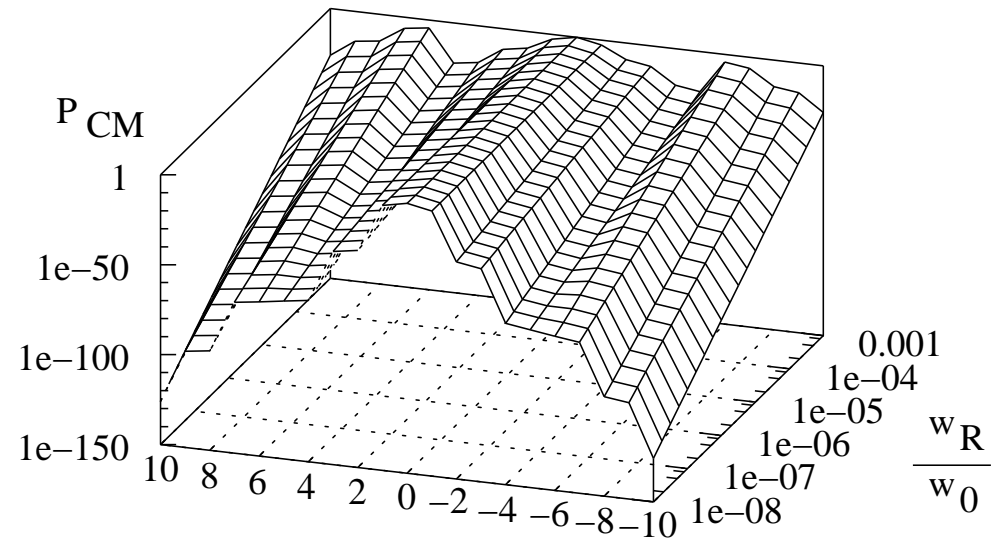

LG winding number 1

Figure 2. The CM transition probability $P_{C M}$ versus the winding number $l$ and the ratio $w_{R} / w_{0}$. The initial state is characterized by $N_{i}=6$ and $M_{i}=0$, and the final state by $N_{f}=12$ and $M_{f}=\operatorname{sgn}(l)\left(|l|-l^{\prime}\right)$. The values were normalized to $P_{C M}$ for $l=0$.

dependence of the transition probability $P_{C M}$ on the ratio $w_{R} / w_{0}$ will be of the form $P_{C M} \propto\left(w_{R} / w_{0}\right)^{|l|-l^{\prime}}$. This behavior characterizes the approximation $w_{R}$ much smaller than $w_{0}$, and one may compare it with the dependence obtained from formulas derived in Ref. [7 in order to establish the validity range of the approximation.

\section{Conclusions}

The selection rules and the matrix elements (13) shows that dipole transitions affect only the CM transition. On the contrary, for quadrupole transitions there is an exchange of one unit of angular momentum between the LG beam and the electronic states, and no limitation exists in the exchange between the $\mathrm{CM}$ and angular momentum of the beam [see first bracket in (13)]. In the case of an atomic system trapped in an harmonic potential the dependence of the $\mathrm{CM}$ transition (selection rules and transition probability) on the LG winding number $l$ is discussed. There are systems [15] like silver nanoparticle where the quadrupole transition are even more intense than dipolar transitions, so that the difference of about two orders of magnitude between the electronic dipole and quadrupole transition can be decreased. In this case we suggest to perform absorption experiment using LG beam to test the dependence of quadrupole transition on $l$. Very likely, due to the correspondence of quadrupole transformation 
rules and polarizability tensor, the dependence of CM motion on $l$, found in this paper and in Ref. [7, would affect Raman and Brillouin transition intensity and the relative selection rules. This would further increase the interest in using LG beam for novel spectroscopy studies. The application of LG beams to Raman and Brillouin transitions will be investigated in a further work. We conclude pointing out that even if the internal transition in quadrupole approximation is limited to one unit of OAM, the dependence of $\mathrm{CM}$ transition on $l$ is of interest in non resonant transition, where the angular momentum of the LG beam, can be used to modulate the transition probability.

\section{References}

[1] Allen L, Beijesbergen M W, Spreeuw R J C and Woerdman J P O 1992 Phys. Rev. A 458185

[2] Allen L, Barnett S M and Padgett M J 2003 Optical Angular Momentum (Institute of Physics Publishing, Bristol and Philadelphia)

[3] Mair A, Vaziri A, Weihs G and Zeilinger A 2001 Nature (London) 412313

[4] Allen L, Babiker M, Lai W K and Lembessis W E 1996 Phys. Rev. A 544259

[5] Tabosa J W R and Petrov D V Phys. Rev. Lett. 834967

[6] Muthufrishnan A and Stroud Jr C R 2002 J. Opt. B: Quantum Semiclass. Opt. 4 S73-S77

[7] Babiker M, Bennett C R, Andrews D L and Dávila Romero L C 2002 Phys. Rev. Lett. 89143601

[8] van Enk S J 1994 Quantum Opt. 6 445-457

[9] van Gelderen M 1998 DEOS Progress Letters 98.1 57-67

[10] Lembessis V E, Babiker M, Baxter C and Loudon R 1993 Phys. Rev. A 481594

[11] Berry M V in Orbital Angular Momentum, edited by Allen L, Barnett S M and Padgett M J 2003 (Institute of Physics Publishing, Bristol and Philadelphia)

[12] Jáuregui R 2004 LANL e-print quant-ph/0404019

[13] Prudnikov A P, Brychkov Yu A and Marichev O T 1992 Integrals ans Series 3rd edn, vol 1-3 (New York: Gordon and Breach)

[14] Molina Terriza G, Torres J P and Torner L 2002 Phys. Rev. Lett. 88013601

[15] Chumanov G, Sokolov K and Cotton T M 1996 J. Phys. Chem. US 100 (13) 5166 